\title{
Treatment Outcome in Patients with Metastatic Non-Small Cell Lung Cancer at Presentation (Retrospective Study)
}

\author{
Abd El-Monaem Mohamed Abd El-Monaem ${ }^{1, *}$ MSc, Wael Helmy EL-Sheshtawy ${ }^{1}$ MD, Mohsen Salah El Din \\ Zekry $^{1}$ MD
}

*Corresponding Author:

Abd El-Monaem Mohamed menaem90@gmail.com

Received for publication January 20, 2020; accepted January 24, 2020; published on line January 26, 2020.

Copyright 2020 The Authors published by Al-Azhar University, Faculty of Medicine, Cairo, Egypt. All rights reserved. This an openaccess article distributed under the legal terms, where it is permissible to download and share the work provided it is properly cited. The work cannot be changed in any way or used commercially.

doi:10.21608/aimj.2020.22738. 1093

${ }^{1}$ Clinical Oncology \& Nuclear Medicine Department, Faculty of Medicine, Al-Azhar University, Cairo Egypt.

\begin{abstract}
Background: worldwide, lung cancer representing 13\% of all new cancer cases And has the highest mortality rate. In Egypt, lung cancer incidence is about $4.6 \%$ of all cancers. recent trials reporting that immune therapy (anti PDL1) showed a significant superior progressionfree survival (PFS) and overall survival (OS) if compared with standard chemotherapy.

Objectives: is to assess outcome of treatment in patients with metastatic non-small cell lung cancer at presentation, in term of response to treatment, progression free survival and, overall survival, treatment elated toxicity.

Patients and Methods: patient related data: Age, sex, special habits, and performance status. Disease related data: date of diagnosis, extent of disease, histopathology details and sites of metastasis, Treatment related data:- Chemotherapy (regimen, cycles, response related toxicity), Radiotherapy (site, related toxicity ), Response to treatment according to RECIST criteria .Progression free survival, Overall survival Results: our study among 56 patients, 29 patients received chemotherapy. radiotherapy used for palliation of symptoms, patients who received chemotherapy showed improvement of progression free survival and overall survival when compared with those who didn't receive chemotherapy, there were no available data about quality of life, because of unavailability of resources we didn't use targeted or immune therapies

Conclusion: patients with metastatic NSCLC, if molecular tests of gene mutations and checkpoints expressions not available, a four to six cycles of platinum based chemotherapy is indicated in patients with good performance status as regard that chemotherapy showed survival benefit versus best supportive care.
\end{abstract}

Keywords: Metastatic; Lung; Cancer

\section{INTRODUCTION}

Worldwide, lung cancer occurred in approximately 1.8 million patients in 2012 representing $13 \%$ of all new cancer cases) and caused an estimated 1.6 million deaths, lung cancer has the highest mortality rate worldwide.$^{6}$ In the United States, there will be approximately 234,000 new cases of lung cancer and over 154,000 deaths annually. ${ }^{18}$

In Egypt , The lung cancer incidence is about $4.6 \%$ of all cancers in both sexes, representing about $6.9 \%$ of male cancers (5017 cases) and $2.5 \%$ of female cancers (3634 cases) and mortality rate $6.8 \%$ in males and $3.8 \%$ in females (Globocan fact sheet, Egypt 2012).Lung cancer deaths in Egypt reached 4,429 per 100,000 of population in 2002.3

Platinum-based chemotherapy remains the standardof-care for most patients affected by advanced non small cell lung cancer (NSCLC), Recent evidence emphasized that the optimal number of first-line platinum cycles should be four for any NSCLC histology. ${ }^{3}$
Authorship: All authors have a substantial contributions to the article.

Disclosure: The authors have no financial interest to declare in relation to the content of this article. The Article Processing Charge was paid for by the authors.

After the era of advances in the molecular biology, many mutations had been discovered in lung cancers

cells, these mutations are currently drive the choice of first line treatment targeted therapy in such patients after the statistical significant survival and clinical benefits with high acceptable toxicity profile that had observed with the use of this class of therapy when compared with chemotherapy as first line treatment ${ }^{17}$

Recently, the use of immune therapy in the first line treatment either alone or in combination with chemotherapy in selected patients with PDL-1 expression showed a significant superior progression-free survival (PFS) and OS when compared with standard chemotherapy alone. Therefore, the Key-note 024 trial was prematurely stopped in order to allow patients receiving 
chemotherapy to cross over to the immunotherapy arm. $^{13}$

The primary objective of this study is to assess the outcome of treatment in patients who presented with metastatic non-small cell lung cancer in term of response to treatment, treatment related toxicity, progression free survival and overall survival.

\section{PATIENT AND METHODS}

This Retrospective study included 56 patients with metastatic non-small cell lung cancer who received systemic treatment at our department during the period between June 1st 2012 and June 1st 2017, and meet the following inclusion criteria; histopathology confirming non-small cell lung cancer either histologically or cytological, age younger than 80 years old, Performance status 0-3 (WHO) and radiological confirmed distant metastases at presentation.

Charts of included patients have been retrieved from the archive to collect the following data: Age, sex, special habits (eg. smoking)، comorbidity, performance status, histopathology, chemotherapy related details, radiotherapy if received, and finally response to treatment according to RECIST criteria.

Overall survival interval was considered as the period between the date of histological diagnosis and the date of the last follow-up (for censored observations) or the date of death (for uncensored observations), while progression free interval was considered as the period between date of the first treatment and the date of the last follow-up (for censored observations), or date of death or disease progression whichever happen first (for uncensored observations).

\section{Statistical methods:}

Data were coded and entered using the statistical package SPSS version 23. Data was summarized using mean, standard deviation, median, minimum and maximum for quantitative variables and frequencies (number of cases) and relative frequencies (percentages) for categorical variables. One-sided log-rank of Kaplan-Meier survival estimates was used for statistical analysis of overall survival and progression free survival, while the unpaired $\mathrm{T}$ test and one-way ANOVA test were used in the univariate analysis of the variables. Results of P-values less than 0.05 were considered as statistically significant.

\section{Ethical approval:}

The current investigation was had been executed based on the recommendations approved by the ethical committee, Faculty of Medicine, Al-Azhar University, Cairo, Egypt, before the start of this study.

\section{RESULTS}

In the study group, the mean age of the 56 included patients was $57.9( \pm 10.3)$, male patients were 44
(78.6\%), and females were $12(21.4 \%)$, with male to female ratio $3.5: 1$ respectively; 40 patients $(71.4$ $\%)$ were smokers and 16 patients $(28.6 \%)$ weren't smokers; performance status (PS) at presentation was reported 0 for 23 patients (41.1\%), PS I for 18 patients (32.1\%), PS II for 12 patients $(21.4 \%)$ and PS III for three patients $(5.4 \%)$

All patients had NSCLC with different subtypes: 35 patients $(62.5 \%)$ had adenocarcinoma, nine patients $(16.07 \%)$ had squamous cell carcinoma, seven patients $(12.5 \%)$ had undifferentiated carcinoma, four patients $(7.14 \%)$ hadlarge cell carcinoma and one patient only $(1.78 \%)$ had spindle cell (sarcomatoid); as regard pathological grading, 30 Patients (54.5\%) had grade II and 26 patients $(45.5 \%)$ had grade 3 tumors; the most common site of metastasis was bone in 21 patients (37.5\%) followed by contralateral lung or pleural metastasis in 18 patients $(32.1 \%)$, suprarenal metastasis in 14 patients $(25.0 \%)$, brain metastasis in 13 patients $(23.2 \%)$ and liver metastasis 6 patients $(10.7 \%)$. Table (1)

Active treatment was delivered for 43 patients (76.8\%) while 13 patients $(23.2 \%)$ didn't receive active treatment; 29 patients $(51.7 \%)$ received chemotherapy with a median number of four cycles (range 1-9); 21 patients $(37.5 \%)$ received Gemcitabine + cisplatin, five patients (8.9\%) received carboplatin + etoposide, tow patients (3.6 $\%)$ received paclitaxel + cisplatin and one patient $(1.7 \%)$ received carboplatin + paclitaxel; while 37 patients $(65.9 \%)$ received palliative radiotherapy of them 14 patients $(25 \%)$ missed after end of treatment. On the other hand six patients $(10.7 \%)$ missed after first visit, seven patients (12.5\%) managed with best supportive care.

Only 7 patients $(12.5 \%)$ was able to receive second line chemotherapy: six of them $(10.7 \%)$ received taxenes based regimen (paclitaxel or docetaxel) and only one patient $(1.7 \%)$ received (cisplatin + navelbine).

The most common toxicities showed with chemotherapy :grade $2-3$ Anemia in 15 patients (51\%) who treated with (cisplatin $\backslash$ gemcitabine) or (carboplatin \gemcitabine) regimens, G2-3 Nausea and vomiting developed in 14 patients $(48.2 \%)$ who received platinum based chemotherapy, however some patients developed another toxicity profiles either related to chemotherapy or radiotherapy but it was insignificant as showed in table (2)

According to RECIST criteria v1.1 response to 1 st line chemotherapy was reported in patients charts as following; 11 patients $(19.6 \%)$ had partial response, four patients $(7.1 \%)$ had stationary disease and 11 patients ( $19.6 \%$ ) developed disease progression, while no assessment was reported in the charts of three patients (5.35\%). table (3): 


\begin{tabular}{|c|c|c|c|}
\hline \multirow{3}{*}{ Gender } & Sex & Count & $\%$ \\
\hline & Female & 12 & 21.4 \\
\hline & Male & 44 & 78.6 \\
\hline \multirow[t]{2}{*}{ Age (yrs.) } & \multicolumn{2}{|l|}{ Mean \pm SD } & Range \\
\hline & \multicolumn{2}{|l|}{$57.9 \pm 10.3$} & $31-76$ \\
\hline P.S (Who) & \multicolumn{2}{|l|}{ Count } & $\%$ \\
\hline $\mathbf{0}$ & \multicolumn{2}{|l|}{3} & 5.4 \\
\hline I & \multicolumn{2}{|l|}{23} & 41.1 \\
\hline II & \multicolumn{2}{|l|}{18} & 32.1 \\
\hline III & \multicolumn{2}{|l|}{12} & 21.4 \\
\hline Median (range) & \multicolumn{3}{|l|}{ II ( 0 -III ) } \\
\hline \multirow{3}{*}{ Pathological differentiation } & Grade & Count & $\%$ \\
\hline & II & 30 & 54.5 \\
\hline & III & 26 & 45.5 \\
\hline \multirow{6}{*}{ Histopathology } & Type & Count & $\%$ \\
\hline & Adenocarcinoma & 35 & 62.5 \\
\hline & Squamous cell & 9 & 16.07 \\
\hline & Undifferentiated carcinoma & 7 & 12.5 \\
\hline & Large cell carcinoma & 4 & 7.14 \\
\hline & Spindle cell ( sarcomatoid ) & 1 & 1.78 \\
\hline \multirow{6}{*}{ Site of Metastasis } & Site & Count & $\%$ \\
\hline & Suprarenal & 14 & 25 \\
\hline & Brain & 13 & 23.2 \\
\hline & Bone & 21 & 37.5 \\
\hline & Contralateral Lung and $\backslash$ or Pleura & 18 & 32.1 \\
\hline & Liver & 6 & 10.7 \\
\hline
\end{tabular}

Table 1: Patients and disease criteria 


\begin{tabular}{|c|c|c|c|}
\hline Treatment lines & Count & \multicolumn{2}{|c|}{$\%$} \\
\hline Chemotherapy $1^{\text {st }}$ line & 29 & \multicolumn{2}{|c|}{51.7} \\
\hline Chemotherap2 $^{\text {nd }}$ line & 7 & \multicolumn{2}{|c|}{12.5} \\
\hline palliative radiotherapy & 37 & \multicolumn{2}{|c|}{65.9} \\
\hline Site of palliative RTH & Count & \multicolumn{2}{|c|}{$\%$} \\
\hline Whole brain RTH & 13 & \multicolumn{2}{|c|}{23.1} \\
\hline Bone & 18 & \multicolumn{2}{|c|}{32.07} \\
\hline Localized field brain RTH & 1 & \multicolumn{2}{|c|}{1.7} \\
\hline Mediastinum & 2 & \multicolumn{2}{|c|}{3.4} \\
\hline Hemostatic RTH to lung & 3 & \multicolumn{2}{|c|}{5.35} \\
\hline Total No & 37 & \multicolumn{2}{|c|}{65.9} \\
\hline & Chemotherapy regimen & Count & $\%$ \\
\hline \multirow{4}{*}{$\mathbf{1}^{\text {st }}$ line chemotherapy } & carboplatin + taxol & 1 & 1.7 \\
\hline & carboplatin + vepsid & 5 & 8.9 \\
\hline & Gemzar + platinol & 21 & 37.5 \\
\hline & taxol + platinol & 2 & 3.6 \\
\hline \multicolumn{2}{|c|}{ Total No of patients with $1^{\text {st }}$ line chemotherapy } & 29 & 51.7 \\
\hline No of cycles & Median (range) & \multicolumn{2}{|c|}{$4(1-9)$} \\
\hline \multirow{3}{*}{$2^{\text {nd }}$ line chemotherapy } & Regimen & Count & $\%$ \\
\hline & Taxenes & 6 & 10.7 \\
\hline & Cisplatin $\backslash$ navelbine & 1 & 1.7 \\
\hline \multicolumn{2}{|c|}{ Total No. of patients with $2^{\text {nd }}$ line chemotherapy } & 7 & 12.5 \\
\hline \multirow{4}{*}{ Hematology } & Toxicity & Count & $\%$ \\
\hline & Neutropenaia & 5 & 17 \\
\hline & Anemia & 15 & 51 \\
\hline & Thrombocytopenia & 2 & 6.8 \\
\hline \multirow{4}{*}{ GIT } & toxicity & Count & $\%$ \\
\hline & Mucositis & 2 & 6.8 \\
\hline & Nausea and Vomiting & 14 & 48.2 \\
\hline & Diarrhea & 3 & 10 \\
\hline \multirow{4}{*}{ RTH toxicity } & toxicity & Count & $\%$ \\
\hline & No & 22 & 78.6 \\
\hline & Yes & 7 & 21.4 \\
\hline & Total & 29 & 100 \\
\hline
\end{tabular}

Table 2: Treatment received and toxicity profile

\begin{tabular}{|l|c|c|c|}
\hline \multirow{2}{*}{ Response criteria } & Category & Count & $\%$ \\
\hline \multirow{3}{*}{$($ RECIST) criteria } & disease progression & 11 & $19.60 \%$ \\
\cline { 2 - 4 } & no assessment & 3 & $5.35 \%$ \\
\cline { 2 - 4 } & Partial response & 4 & 11 \\
\cline { 2 - 4 } & stationary course & $4.60 \%$ \\
\hline
\end{tabular}

Table 3: Response to treatment

The median progression free survival was 8.9 months for all patients included in this study, however it was 9.3 and 7.5 months for patients who received chemotherapy and didn't receive any treatment respectively, with no statistical difference between the two group $(\mathrm{P}=0.3)$. Figure (1) 


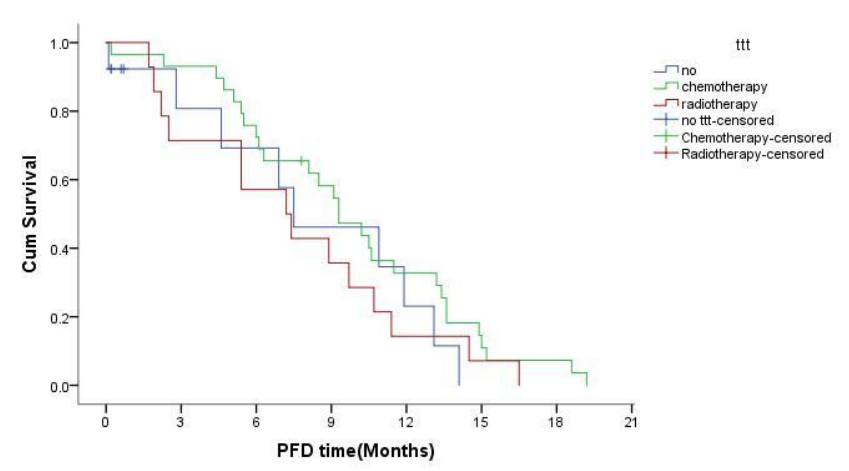

Fig. 1: Median Progression free survival (PFS) curve

Median overall survival (MOS) was 10.8 months, at subgroup analysis systemic treatment showed significant improving in overall survival as following : in patients who didn't receive treatment MOS 10.8 but in patients who received chemotherapy MOS was $13.6(9.3-17.9)(\mathrm{P}=0.019)$. Figure (2)

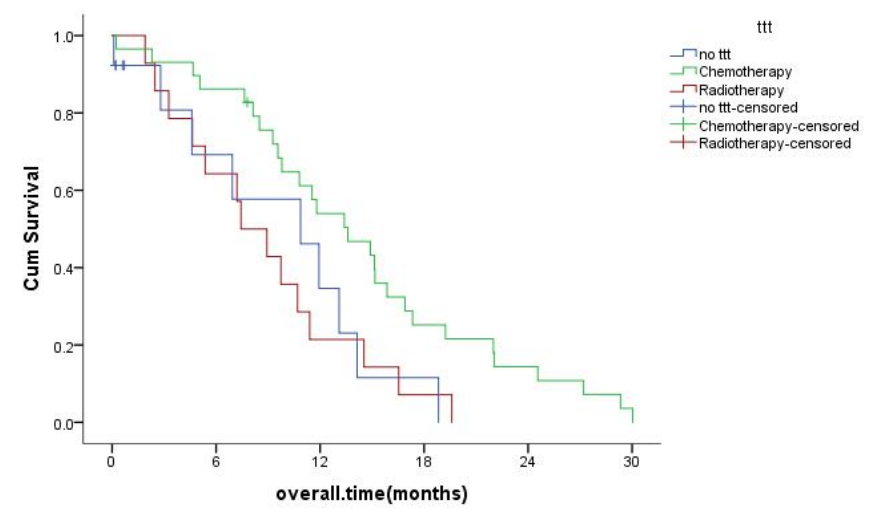

Fig. 2: median overall survival (MOS) curve

On the other hand PFS and OS did not significantly affected by any of the following factors; age, sex, PS, special habits, histopathological subtype, pathological grade, site of metastasis, type of chemotherapy or number of cycles.

\section{DISCUSSION}

Worldwide, lung cancer occurred in approximately 1.8 million patients representing $13 \%$ of all new cancer cases and caused an around 1.6 million deaths, lung cancer has the highest mortality ${ }^{6}$

In the five years duration of this study the total number of patients who presented to our department were 4636 , patients who diagnosed with lung cancer were 152 cases $(3.27 \%)$, total number who presented with metastatic Non-small cell lung cancer were 61 (40\% of total lung cancer patients), so in our department, incidence of lung cancer is different from international incidence. Where in Egypt incidence of lung cancer in the period between 2008 and 2011 was $4.22 \%$ of all ${ }^{3}$
In our study the mean age was 58 years and male to female ratio was $3.5: 1$ which is different from the published data by Brule, where he reported mean age of 67 years and male to female ratio $1.2: 1$ (Stephanie Y. Brule; et.al 2016), this difference could explained by the higher rate of smokers among Egyptian males and lower rate of smokers among Egyptian females.

According to the current treatment guidelines by NCCN and ESMO, all patients who are newly diagnosed with stage IVNSCLC and has none squamous histology or have squamous histology based on small biopsy or nonsmoker should be tested by broad molecular profiling, and 1st line treatment should be based on the result of this testing. ${ }^{12}$ 
Up to this moment the targeted therapy not provided by the Egyptian health ministry to none insured patients, that represent the majority patients receiving treatment at our department, because of this the overall survival in the patients included in our study is considered very low compared to the published data in the era of targeted therapy ${ }^{19}$

With stage IV disease who have a good PS benefit from chemotherapy, usually with a platinum-based regimen is recommended for patients with stage IV NSCLC and negative or unknown test results for ALK rearrangements or sensitizing EGFR mutations, PD-L1 expression less than $1 \%$ or unknown and contraindications to immunotherapy. Recommended chemotherapy regimens are based on PS and include platinum agents (eg, cisplatin carboplatin), taxanes (eg, paclitaxel, albumin-bound paclitaxel [also known as nab-paclitaxel], docetaxel), vinorelbine, etoposide, pemetrexed, and gemcitabine ${ }^{4}$

Patients included in our study received platinum based chemotherapy mean No of cycles were 4 range (1-9) and radiotherapy used as palliative treatment for symptomatic patients that similar to published data reported that Chemotherapy improves both survival and quality of life in metastatic NSCLC, four cycles of platinum based Chemotherapy (max 6 cycles) is the standard treatment for patient with good Performance status. Patients with border line Performance status $=2$ may benefit from single agent. Palliative radiotherapy is introduced for symptomatic patients only ${ }^{22}$, Four to six cycles of platinum based chemotherapy is indicated in patients with metastatic non small cell lung cancer with good performance status, Extending the duration of treatment with the initial platinum based chemotherapy beyond four to six cycles is not recommended ${ }^{4}$

In our study we considered grades 2-4 toxicities of chemotherapy among who received chemotherapy , 15 patients (51\%) developed Anemia with cisplatin $\backslash$ gemcitabine or carboplatin \ gemcitabine regimens, 14 patients (48\%) developed Nausea and vomiting in platinum containing chemotherapy, 5 patients $(17 \%)$ developed Neutropenia with cisplatin containing regimens , 2 patients $(6 \%)$ developed G4 Thrombocytopenia with cisplatinlcarboplatin + gemcitabine regimens, 2 patients $(6 \%)$ developed Mucositis G2 with carboplatin containing chemotherapy, 3 patients (10 $\%$ ) developed Diarrhea in cisplatin + gemcitabine regimens, which is similar to worldwide publication. ${ }^{4}$

Radiotherapy toxicity profile: 29 patients received palliative Rth ,3 of them complained bony pain at site of Rth over bony Mets, 1 patient complained headache after he finished WBI, 2 pts complained hair loss after WBI, 1 patient complained mucositis and dysphagia G2 after he finished palliative Rth to mediastinal mass. This meets previous publications of reported radiotherapy toxicity by. ${ }^{8}$
In our study group the progression free survival (PFS) had no significant difference between patients who received chemotherapy and those who didn't receive any treatment median PFS was 9.3 months (7.1-11.4 ) for patients who received chemotherapy and 7.5 months $(1.8-13.1)(\mathrm{P}=0.349)$ for patients who didn't receive any treatment that was different with data published by ${ }^{14}$

Unfortunately none of our patients received pemetrexed in their treatment due to unavailability of treatment,that's why we couldn't assess survival on adding pemetrexed to chemotherapy regimens , on the other hand A phase III trial in which Survival in the 847 patients with adenocarcinoma was significantly prolonged with cisplatin plus pemetrexed compared with cisplatin plus gemcitabine (median 12.6 versus 10.9 months respectively). ${ }^{21}$

In our study group median OS was 10.8(3.7-17.9) months in patients didn't receive active treatment versus 13.6 months (9.3-17.9) in patients received chemotherapy ( $p$ value 0.019 ) that was similar to results of data published in(2001) ${ }^{1}$ who reported median OS was ( 13.7 months ) (range 7.4 - 213 weeks $) \quad(p=0.0018)$.in patients who received chemotherapy), and different from results of study published in $2016 .{ }^{20}$ when Median OS was shorter amongst untreated patients (3.9 vs. 10.7 months in Systemic chemotherapy , ( HR 1.80 [95\% CI 1.42.3], $\mathrm{p}<0.01)$.

The use of immune therapy in the first line treatment either alone or in combination with chemotherapy in selected patients with PDL-1 expression showed a significant superior progression-free survival (PFS) and OS when compared with standard chemotherapy alone. Therefore, according to ESMO guidelines patients of (KEYNOTE-024 trial was prematurely stopped receiving chemotherapy to cross over to the immunotherapy arm . ${ }^{13}$

Unfortunately Up to this moment immune therapy not provided by the Egyptian health ministry to none insured patients, that represent the majority patients receiving treatment at our department so our patients couldn't gain benefit of such treatment option .

\section{CONCLUSION}

In patients with metastatic NSCLC, if molecular tests of gene mutations and checkpoints expressions not available, a four to six cycles of platinum based chemotherapy is indicated in patients with good performance status as regard that chemotherapy showed survival benefit versus best supportive care. 


\section{REFERENCES}

1. Anelli A, Lima A, Younes N, et al. Revista do Hospital das Clínicas Brazil 2001 region (Mucci LA, Hjelmborg JB, Harris JR et al. Familial risk and heritabilityof cancer among twins in Nordic Countries. JAMA 2016; 315: $68-76$.

2. Antonio R. and Massimo DM. Platinumbased chemotherapy in advanced non-smallcell lung cancer: optimal number of treatment cycles, Expert Review of Anticancer Therapy 2016 Jun;16(6):653-60.

3. Amal S. Ibrahim, Hussein M. Khaled, and HossamKamel et.al .Cancer Incidence in Egypt: Results of the National PopulationBased Cancer Registry Program 2011. https://doi.org/10.1155/2014/437971

4. Azzoli G, Kris G and Pfister G. Cisplatin versus carboplatin for patients with metastatic non-small-cell lung cancer--an old rivalry renewed. J Natl Cancer Inst 2007; 99:828.

5. Belani CP, Choy $\mathrm{H}$, Bonomi $\mathrm{P}$, et al. Combined chemoradiotherapy regimens of paclitaxel and carboplatin for locally advanced NSCLC: a randomized phase II locally advanced multi-modality protocol (LAMP). J ClinOncol. 2006; 23:5883-91.

6. Brambilla E, Travis WD. Lung cancer. In: World Cancer Report, Stewart BW, Wild CP (Eds), World Health Organization, Lyon 2014.

https://www.drugsandalcohol.ie/28525/1/Wor ld\%20Cancer\%20Report.pdf

7. Brule $\mathrm{Y}$, Al-Baimani $\mathrm{K}$, Jonker $\mathrm{H}$, et al. Palliative systemic therapy for advanced nonsmall cell lung cancer: investigating disparities between patients who are treated versus those who are not. Lung Cancer. 2016; 97:15-21.

8. Diana F, Franzen A, Menon $\mathrm{R}$, et al. Rationale for treatment of metastatic squamous cell carcinoma of the lung using fibroblast growth factor receptor inhibitors. Chest. 2012; 142(4):1020-6.

DOI:10.1378/chest.11-2943

9. Griesinger F, Krefting F, Basara $\mathrm{N}$, et al. Clinical Experience of Immunotherapy Treatment: Efficacy and Toxicity Analysis of the Compassionate Use Program of Nivolumab in Patients with Advanced Squamous Cell Non-Small Cell Lung Cancer. Oncology research and treatment. 2019; 42(5):243-55.

10. Ibrahim $\mathrm{S}$, Seif-Eldein $\mathrm{A}$, Ismail $\mathrm{K}$ et al. "Cancer in Egypt, Gharbiah," Triennial Report of 2000-2002, Gharbiah PopulationBased Cancer Registry, Middle East Cancer Consortium, Cairo, Egypt, 2014. https://doi.org/10.1155/2014/437971
11. Kivrak S, Göktürk $\mathrm{T}$, Kivrak $\mathrm{I}$, et al Investigation of phenolic profiles and antioxidant activities of some Salvia species commonly grown in Southwest Anatolia using UPLC-ESI-MS/MS. Food Science and Technology. 2019; 39(2):423-31.

12. Lindeman N, Cagle $\mathrm{P}$, Aisner $\mathrm{D}$ et al. Updated molecular testing guide-line for the selection of lung cancer patients for treatment with targeted tyrosine kinase inhibitors: guideline from the College of American Pathologists, the International Association for the Study of Lung Cancer, and the Association for Molecular Pathology. Arch Pathol Lab Med 2018; 142: 321-346

13. Martin R, Delvys R, Andrew G. et al. updated Analysis of KEYNOTE-024: Pembrolizumab Versus Platinum-Based Chemotherapy for Advanced Non-Small-Cell Lung Cancer With PD-L1 Tumor Proportion Score of 50\% or Greater. doi: $10.1200 / \mathrm{JCO} .18 .00149$

14. Paz-Ares L, Hirsh V, Zhang L. et al. Monotherapy Administration of Sorafenib in patients with non-small cell lung cancer (MISSION) trial: a phase III, multicenter, placebo-controlled trial of Sorafenib in patients with relapsed or refractory predominantly Nonsquamous non-small-cell lung cancer after 2 or 3 previous treatment regimens. $J$ Thorac Oncol. 2016; 10(12):1745-53.

15. Petrosyan L, Johung L, Yeh N, et al. Extended Survival and Prognostic Factors for Patients With ALK-Rearranged Non-SmallCell Lung Cancer and Brain Metastasis. $J$ Clin Oncol 2016. DOI: $\underline{10.1200 / J C O .2015 .62 .0138}$

16. Pijls-Johannesma M, De Ruysscher D and Vansteenkiste J. Timing of chest radiotherapy in patients with limited stage small cell lung cancer: a systematic review and meta-analysis of randomised controlled trials; 33(5):461-73.

17. Rybarczyk-Kasiuchnicz, A. and R. Ramlau,Current views on molecularly targeted therapy for lung cancer - a review of literature from the last five years. Kardiochir Torakochirurgia Pol, 2018. 15(2): p. 119-124.

18. Siegel L, Miller D and Jemal A. Cancer statistics CA Cancer J Clin 2018. DOI: $\underline{10.3322 / \text { caac. } 21442}$

19. Solomon J, Mok T, Kim W et al. First-line crizotinib versus chemo-therapy in ALKpositive lung cancer. $N$ Engl J Med 2014; 371: 2167-77

20. Stephanie Y. Brulea, Khalid Al-Baimani a, Hannah Jonker et al. 2016 Palliative systemic therapy for advanced non-small cell lung cancer: Investigating disparities between 
patients who are treated versus those who are not.

DOI: https://doi.org/10.1016/j.lungcan.2016.0 $\underline{4.007}$

21. Syrigos N, Vansteenkiste J, Parikh P, et al. Prognostic and predictive factors in a randomized phase III trial comparing cisplatin-pemetrexed versus cisplatingemcitabine in advanced non-small-cell lung cancer. Ann Oncol 2010; 21:556.

22. Yorke D, Wang L, Rosenzweig E, et al. Evaluation of deep inspiration breath hold lung treatment plans with Monte Carlo dose calculation, International Journal of Radiation Oncology, Biology and Physics, 2007; 53(4): 1058-70. 